\title{
Comparison of Placido, Scheimpflug and Combined Dual Scheimpflug-Placido Technologies in Evaluating Anterior and Posterior CLMI, SimK's as well as Kmax, in Keratoconic and Postrefractive Surgery Ectasia
}

\author{
Thomas F Mauger, Ashraf M Mahmoud, Cynthia J Roberts, Lena V Chheda, Rebecca A Kuennen
}

Andrew J Hendershot, Richard G Lembach

\begin{abstract}
Purpose: To calculate and compare cone location and magnitude index (CLMI ), Kmax and other corneal measures derived from three different technologies, Placido, Scheimpflug, and a combination dual Scheimpflug-Placido device, from the same group of eyes with keratoconus and postrefractive surgery corneal ectasia.
\end{abstract}

Methods: Keratoconus $(n=26)$ eyes of $(n=19)$ subjects and postrefractive surgery ectasia $(n=5)$ eyes of $(n=5)$ subjects were selected to have measurements performed using the Keratron Scout, Pentacam HR and Galilei Dual Scheimpflug Analyzer. Device-generated SimK's and device-specific CLMI and Kmax indices as well as map data, were exported from each device. Index values for multiple exams were averaged. The map data were processed using The Ohio State University Corneal Topography Tool (OSUCTT) to calculate CLMI parameters, Kmax and SimK values using consistent algorithms on all three devices. Maps were averaged before calculation for multiple examinations. Repeated measures analysis of variance and posthoc analysis were used to identify differences between devices.

Results: The anterior axial CLMI calculated from the Keratron data was significantly higher than CLMI for the Galilei ( $p=$ 0.0443 ) or Pentacam $(p<0.0004)$ with keratoconus, 12.23 compared with 11.20 and 11.00 diopters, respectively. Kmax was also significantly higher in the Keratron than the Galilei $(p=0.0063)$ or the Pentacam $(p<0.0002)$. Galilei and Pentacam were not significantly different from each other in either CLMI $(p=0.6287)$ or $\operatorname{Kmax}(p=0.2115)$. The anterior CLMI values for the postrefractive surgery ectasia eyes were not significantly different between devices. Posterior CLMI values were calculated from the Galilei and Pentacam data and were -2.60 and -2.46 diopters $(p=0.1173)$ for keratoconus and -2.66 and -3.04 diopters $(p=0.2242)$ for postrefractive surgery ectasia.

Conclusion: The small cone Placido measured dioptric values that were greater than the pure Scheimpflug system, but the difference was only about 1 diopter, which is not relevant clinically in evaluating and managing ectasia. The combined dual Scheimpflug-Placido system produced measured dioptric values between the other two technologies. The anterior CLMI calculations accurately predicted keratoconus with all three devices. The posterior CLMI in ectasia may be a potentially valuable calculation in demonstrating asymmetric steepening.

Keywords: Keratoconus, Collagen corneal cross-linking (CXL), Corneal topography, Placido disk topography, Ectasias.

How to cite this article: Mauger TF, Mahmoud AM, Roberts CJ, Chheda LV, Kuennen RA, Hendershot AJ, Lembach RG. Comparison of Placido, Scheimpflug and Combined Dual Scheimpflug-Placido Technologies in Evaluating Anterior and Posterior CLMI, SimK's as well as Kmax, in Keratoconic and Postrefractive Surgery Ectasia. Int J Keratoco Ectatic Corneal Dis 2012;1(1):44-52.
Source of support: Clinical Research Consultants, Inc., Topcon Medical Systems, Inc., and SOOFT Italia, Ohio Lions Eye Research Foundation.

\section{Conflict of interest:}

- C Roberts is a Consultant for Oculus Optikgerate $\mathrm{GmbH}$ and Ziemer Ophthalmic Systems AG, and has an interest in the GALILEI.

- A Mahmoud has an interest in the GALILEI.

- T Mauger, L Chheda, R Kuennen, A Hendershot, and $\mathrm{R}$ Lembach have no financial interests.

\section{INTRODUCTION}

Keratoconus is a noninflammatory corneal disorder with thinning and irregular astigmatism. ${ }^{1-3}$ Postrefractive surgery corneal ectasia presents with a similar topographic/ tomographic appearance following refractive surgery. ${ }^{4-7}$ The diagnosis and measurement of these diseases has been advanced by the development of corneal imaging instruments including Placido disk videokeratoscopy and Scheimpflug tomographers. ${ }^{8-11}$ Topographical indices have been developed to detect keratoconus for individual devices. ${ }^{12-19}$ The cone location and magnitude index (CLMI) has been developed for the detection and quantification of keratoconus and can be calculated using data from multiple devices. ${ }^{12-15}$ Placido disk topographic and Scheimpflug tomographic instruments have been compared in normal eyes. ${ }^{11}$ The purpose of this study is to compare CLMI, Kmax and other corneal measures derived from three different technologies, one Placido, one pure Scheimpflug, and one a combination of Placido and Scheimpflug, using the same group of eyes with keratoconic and postrefractive corneal ectasia.

\section{METHODS}

\section{Subjects}

A total of 26 eyes of 19 subjects with kerataconus and five eyes of five subjects with postrefractive surgery ectasia were enrolled. All subjects were originally recruited for an ongoing, industry-sponsored FDA clinical trial of corneal collagen cross-linking. Scheimpflug tomography was measured using the Pentacam HR (Oculus, Wetzler, Germany). Additional diagnostic devices were added to the protocol at The Ohio State University site. Placido topography was performed with the Keratron Scout 
topographer (Optikon, Rome, Italy), and the combination of Scheimpflug and Placido tomography/topography was performed with the Galilei Dual Scheimpflug Analyzer (Ziemer, Port, Switzerland). In the current study, the screening examinations were utilized for comparison of topographic and tomographic technologies, with up to three examinations for each subject. Subjects were recruited based on the following inclusion criteria: Diagnosis of keratoconus (minimum age 12 years) with the presence of one or more slit lamp findings, such as scissoring of the retinoscopic reflex, Fleischer ring, Vogt striae or corneal thinning or the diagnosis of postrefractive surgery ectasia (minimum age 18 years); the presence of central or inferior steepening and axial topography consistent with keratoconus or postrefractive corneal ectasia; Kmax value $\geq 47.00 \mathrm{D}$ or I-S ratio $>1.9$ for keratoconus only; BSCVA 20/20 or worse $(<58$ letters on ETDRS chart); and removal of contact lenses for 3 days to 2 weeks prior to the enrollment refraction, depending in the type of lens. Exclusion criteria were corneal pachymetry $\leq 400$ microns at the thinnest point, history of corneal disease (e.g. herpes simplex, herpes zoster keratitis, recurrent erosion syndrome, corneal melt or corneal dystrophy, etc.), clinically significant corneal scarring, history of chemical injury or delayed epithelial healing, pregnancy (including plan to become pregnant) or lactation, known sensitivity to study medications, nystagmus, any condition that would prevent a steady gaze or interfere with or prolong epithelial healing and for keratoconus only, previous corneal surgery or the insertion of Intacs.

\section{Devices}

\section{Pentacam HR}

The Pentacam HR is a high resolution rotating Scheimpflug camera with a blue slit light that successively illuminates a minimum of 25 meridional slits over $360^{\circ}$ through the cornea during each measurement. Software version 1.17 was used in the current study. The Pentacam can be considered a tomographer, since it acquires data on all anterior segment structures, including anterior and posterior cornea surfaces, iris and lens. The precision of SimK measurements with the Pentacam HR has been reported to be good with repeatability limits of 0.25 and 0.36 diopters for $\mathrm{K} 1$ and $\mathrm{K} 2$ respectively. ${ }^{20}$ In separate reports, the coefficient of repeatability for simulated keratometry on the Pentacam was reported to be \pm 0.28 diopters, indicating good repeatability, ${ }^{21}$ and the coefficient of variation was reported to be within $1 \% .{ }^{22}$ No studies of accuracy have been published due to the lack of an appropriate test surface. SimKs and Kmax (maximum curvature on the axial map) were exported for analysis.

\section{Galilei Dual Scheimpflug Analyzer}

The Galilei uses dual rotating Scheimpflug cameras with a blue slit light that successively illuminates a minimum of 15 meridional slits with simultaneous image capture by both opposing cameras over $180^{\circ}$, all integrated with a flat 20-ring, infrared Placido disk topographer which captures two images $90^{\circ}$ apart during each measurement. Software version 5.2.1 was used in the current study. The Galilei can be considered a tomographer/topographer, since it acquires data on all anterior segment structures, including anterior and posterior cornea surfaces, iris and lens as well as integrated Placido topography. Reproducibility of the Galilei reported as intraclass correlation coefficients (ICC) in central corneal thickness, Kflat and Ksteep were 0.98, 0.97 and 0.84 respectively. ${ }^{23}$ In pre- and postrefractive surgery population, ICCs were greater than 0.99 for SimKs, as well as other measures of corneal power and the coefficient of variation was less than $0.5 \%$ in corneal power. ${ }^{24}$ No studies of accuracy have been reported due to the lack of an appropriate test surface. SimKs were exported for analysis.

\section{Keratron Scout}

The Keratron is a small cone, 28-ring, visible light Placido corneal topographer that derives curvature data using an arcstep algorithm to reconstruct 360 meridians through the center and perpendicular to the rings projected onto the cornea. Software version 4.6.6 was used for the current study. Accuracy studies are possible with a Placido topographer due to the existence of test surfaces for comparison of measured to true value. The accuracy of the keratron in the measurement of curvature has been reported between \pm 0.1 and \pm 0.25 diopters. ${ }^{25}$ The repeatability of the keratron in normal subjects has been assessed via coefficient of variation and reported to be within $1 \%{ }^{22}$ The cone location and magnitude index (CLMI) ${ }^{14}$ is calculated on the axial, tangential and Gaussian curvature maps. This index identifies the steepest $2 \mathrm{~mm}$ diameter region within an $8 \mathrm{~mm}$ diameter search zone and compares the average curvature magnitude within this region to a $2 \mathrm{~mm}$ diameter region at the same radial distance from the center in the opposite quadrant. The axial CLMI is used for screening and the tangential curvature CLMI is used to track disease progression. The magnitude of the steepest region and its spatial location are reported by the Keratron for each type of curvature map. For the current study, SimKs and the CLMI index parameters were exported.

\section{Analysis}

Device-generated SimKs and device-specific CLMI and Kmax indices as well as surface data from anterior, posterior 
and pachymetric maps, were exported from each of the three devices. SimKs and index values for multiple examinations were averaged. In order to compare technologies using consistent algorithms, the map data were processed using The Ohio State University Corneal Topography Tool (OSUCTT) and SimKs were calculated, along with the indices, using the same algorithm for each device. ${ }^{12-15}$ Maps were averaged before processing for multiple examinations. CLMI parameters included CLMI on the axial map (CLMI_axi) and the average magnitude of the $2 \mathrm{~mm}$ spot (CLMI_spotaxi) on all devices as well as CLMI on the posterior axial map (PCLMI_axi) and the average magnitude of the $2 \mathrm{~mm}$ spot (PCLMI_spotaxi) on the two tomographers. Kmax was determined by extracting the steepest single value within the $2 \mathrm{~mm}$ CLMI spot on the anterior axial map. Repeated measures analysis of variance and post-hoc analysis were used to compare devices.

SimK, CLMI and Kmax algorithms calculated by the OSUCTT were validated by comparing the native device index against the corresponding calculated index using linear regression analysis. Indices were averaged for multiple maps.

\section{RESULTS}

Validation of the OSUCTT algorithms is shown in Figures 1 to 3 . CLMI and spot parameters from the Keratron compared to OSUCTT CLMI parameters are given in Figure 1. Pentacam-generated Kmax compared to OSUCTTcalculated Kmax is shown in Figure 2, and regression analyses for SimKs by device are shown in Figure 3. For all regressions, $\mathrm{p}$ is $<0.0001$ and $\mathrm{R}^{2}>0.97$.

\section{Keratoconus}

The device-generated and OSUCTT-calculated parameters for the 26 keratoconic eyes are presented in Table 1 and show the p-values produced by the ANOVA to indicate that at least one of the three devices is different. The CLMI_axi data are 11.20, 11.00 and 12.23 diopters respectively for the Galilei, Pentacam and Keratron instruments. The posthoc analysis shows that the Keratron is significantly different from the Galilei in both CLMI_axi $(\mathrm{p}=0.0443)$ and Kmax $(0.0063)$ as well as from the Pentacam in both CLMI_axi $(\mathrm{p}<0.0004)$ and $\operatorname{Kmax}(\mathrm{p}<0.0002)$. However, the Galilei and Pentacam are not significantly different for either CLMI_axi $(p=0.6287)$ or $\operatorname{Kmax}(p=0.2115)$. For CLMI_axi and Kmax as well as CLMI_spotaxi, the Keratron has the highest value. For CLMI_spotaxi, the Keratron was not significantly different from the Galilei $(\mathrm{p}=0.0630)$, but was significantly different from the Pentacam ( $\mathrm{p}=0.0006)$. The Galilei and Pentacam were also significantly different in CLMI_spotaxi $(\mathrm{p}=0.0381)$.
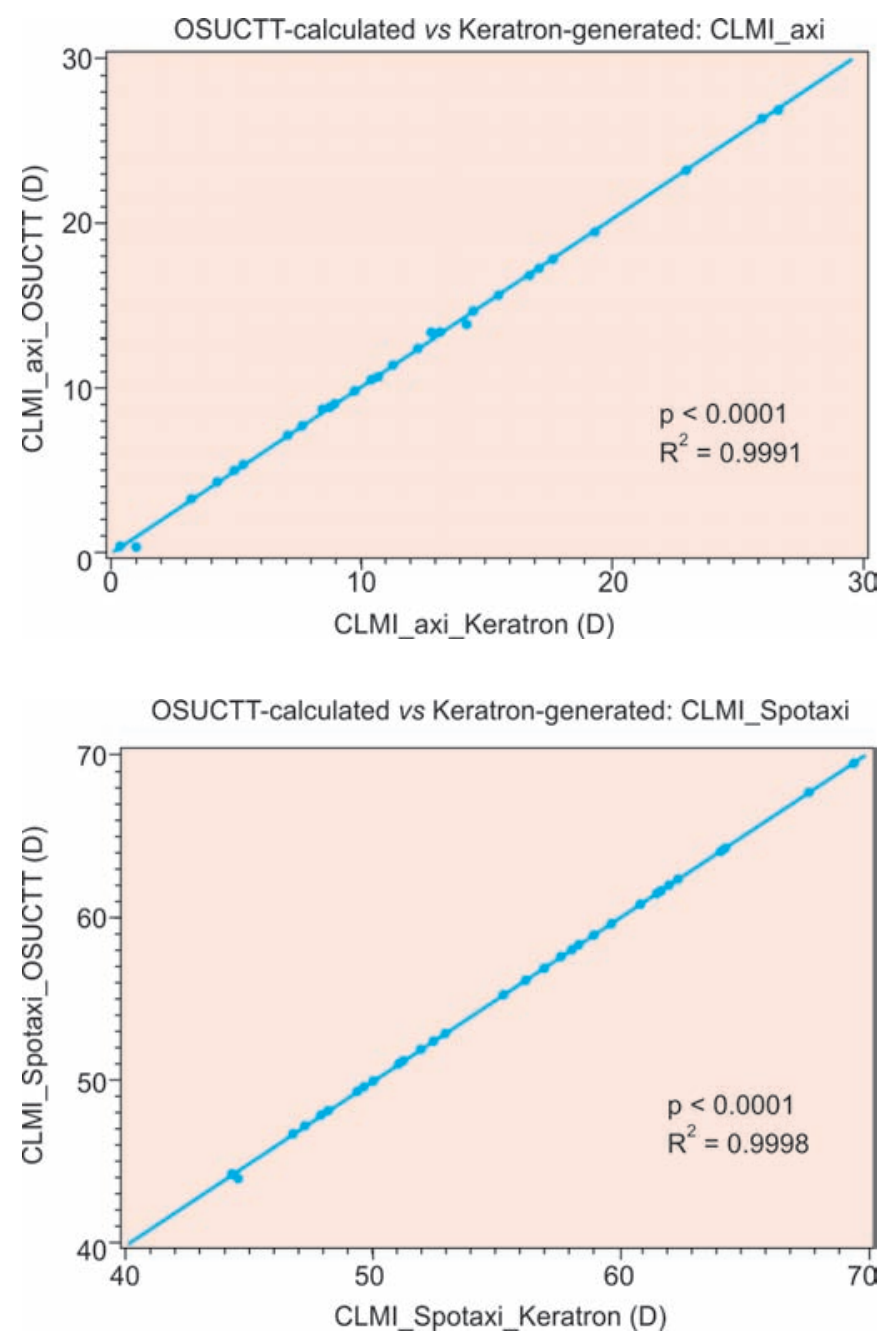

Fig. 1: Regression analyses of CLMI (top) and spot magnitude (bottom) generated by the Keratron compared to CLMI and spot magnitude calculated by OSUCTT using exported data from the Keratron

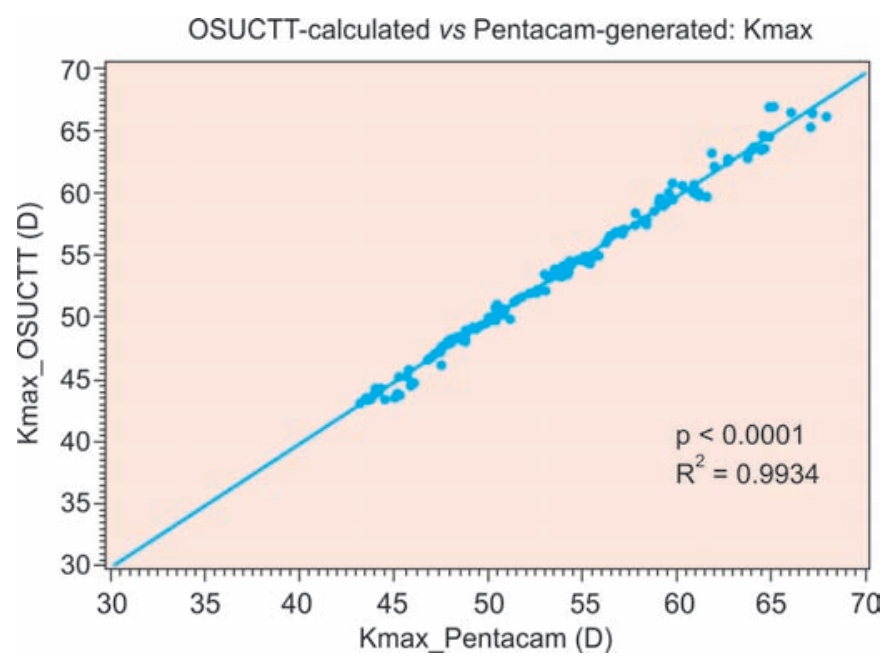

Fig. 2: Regression analysis of Kmax generated by the Pentacam HR compared to Kmax calculated by OSUCTT using exported data from the Pentacam

For the OSUCTT calculated SimK values, the steep meridians were all significantly different from each other $(\mathrm{p}<0.05)$, and none of the flat meridians were different 

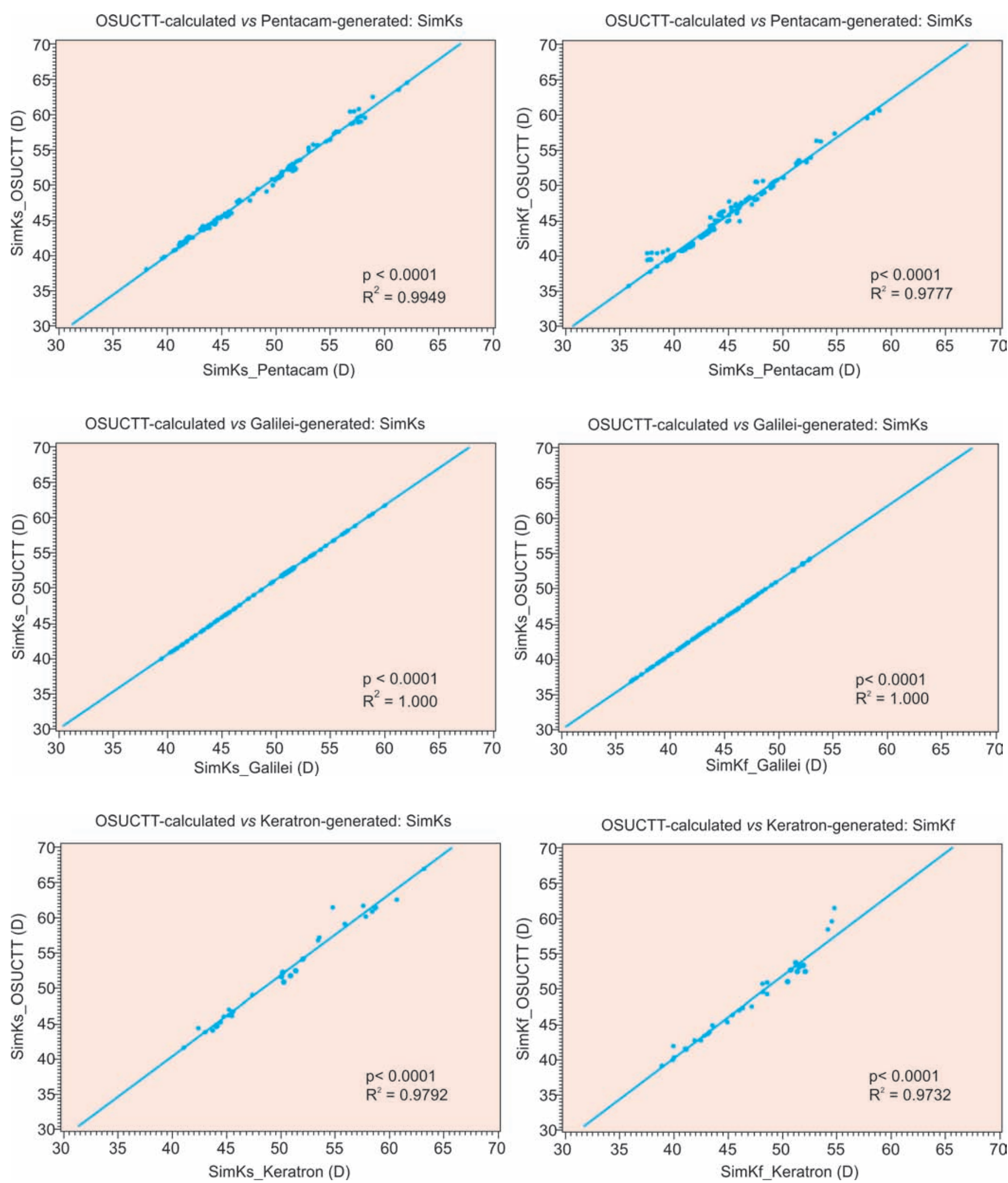

Fig. 3: Regression analyses of Flat SimK (SimKf) and Steep SimK (SimKs) generated by each device compared to SimKs calculated by OSUCTT, with Pentacam on top, Galilei in the middle and Keratron on the bottom

( $p>0.05)$. In the native SimK values, the steep meridian of the Galilei was not significantly different from the Keratron ( $\mathrm{p}=0.4493)$, but was significantly different from the Pentacam ( $\mathrm{p}=0.0001)$ and the Pentacam and Keratron were also significantly different from each other $(\mathrm{p}=0.0043)$.
For the OSUCTT-calculated average SimK, the only two devices that were not significantly different were the Galilei and Pentacam $(p=0.7972)$. The Keratron SimKavg was significantly different from both Pentacam $(\mathrm{p}=0.0015)$ and Galilei $(\mathrm{p}=0.0216)$. For the native SimKavg, however, the 
only two devices not significantly different were the Galilei and Keratron ( $\mathrm{p}=0.8892)$. The Pentacam was significantly different from both the Galilei $(p=0.0077)$ and the Keratron $(\mathrm{p}=0.0139)$.

\section{Ectasia}

The device-generated and OSUCTT-calculated parameters for the five postrefractive surgery ectasia eyes are presented in Table 2. The CLMI_axi data are 12.39, 14.61 and 14.02 diopters for the Galilei, Pentacam and Keratron instruments respectively. They are not significantly different from each other ( $p=0.2024$ ANOVA). Although the ANOVA for Kmax indicates that at least one device is different, none of the individual device comparisons in the post-hoc analysis reaches the 0.05 level of significance. This is likely due to the low number of eyes. None of the other relationships are significantly different.

\section{PCLMI axi}

The CLMI calculated from the posterior corneal axial measurements (PCLMI_axi) from the Galilei and Pentacam instruments was calculated in the same manner as the anterior-based CLMI, and were not significantly different in either group. The measurements were -2.66 and -2.46 diopters for the keratoconic eyes with the Galilei and the Pentacam instruments respectively ( $p=0.1173)$. The measurements were -2.66 and -3.04 diopters respectively, for the postrefractive ectasia eyes $(p=0.2242)$.

\section{DISCUSSION}

A comparison between topographic/tomographic technologies demonstrates that small-cone Placido topography tends to measure the highest dioptric values in the anterior surface parameters, and pure Scheimpflug tomography tends to measure the lowest dioptric values. This is consistent with other reports in the literature in normal eyes. ${ }^{22}$ Combined Placido/Scheimpflug topography/ tomography tended to measure values in between the other two technologies. It is not possible to know which is the most accurate with the current study design, since an accuracy study would require a test surface of known shape. However, it is important to understand how the devices compare in evaluating keratoconus, including screening for

Table 1: Mean values of the parameters in diopters-keratoconic eyes $(\mathrm{N}=26)$

\begin{tabular}{|c|c|c|c|c|}
\hline \multicolumn{5}{|c|}{ Mean (SD) } \\
\hline Parameter & Galilei & Pentacam & Keratron & $\begin{array}{c}p \text {-value (ANOVA) } \\
\text { Significant values in Bold }\end{array}$ \\
\hline Device SimK steep & $49.38(5.53)$ & $48.47(5.14)$ & $49.12(5.57)$ & $<0.0001$ \\
\hline Device SimK flat & $44.61(3.87)$ & $44.43(3.84)$ & 44.78 (3.99) & 0.2870 \\
\hline Device SimK ave & $46.99(4.60)$ & $46.44(4.43)$ & $46.95(4.73)$ & 0.0022 \\
\hline Calc SimK steep & 49.95 (5.65) & $49.25(5.65)$ & $50.52(6.50)$ & $<0.0001$ \\
\hline Calc SimK flat & 44.89 (4.05) & $45.12(4.26)$ & $45.34(4.55)$ & 0.3240 \\
\hline Calc SimK ave & $47.22(4.77)$ & 47.19 (4.91) & $47.93(5.454)$ & 0.0035 \\
\hline Kmax & $54.21(6.29)$ & $54.90(6.14)$ & $56.46(6.68)$ & 0.0007 \\
\hline CLMI_axi & $11.20(5.41)$ & $11.00(5.29)$ & $12.23(5.83)$ & 0.0022 \\
\hline CLMI_Spotaxi & $53.57(5.37)$ & $53.18(5.10)$ & $54.24(5.78)$ & 0.0004 \\
\hline PCLMI_axi & $-2.46(1.07)$ & $-2.60(1.06)$ & & 0.1123 \\
\hline PCLMI-Spotaxi & $-8.27(0.95)$ & $-8.39(1.07)$ & & 0.0192 \\
\hline
\end{tabular}

Table 2: Mean values of the parameters in diopters-postrefractive ectasia eyes $(\mathrm{N}=5)$

\begin{tabular}{|c|c|c|c|c|}
\hline \multicolumn{5}{|c|}{ Mean (SD) } \\
\hline Parameter & Galilei & Pentacam & Keratron & $\begin{array}{c}p \text {-value (ANOVA) } \\
\text { Significant values in Bold }\end{array}$ \\
\hline Device SimK steep & $44.71(4.26)$ & $44.27(3.73)$ & $45.00(4.41)$ & 0.2377 \\
\hline Device SimK flat & $40.65(3.60)$ & $40.57(3.18)$ & $42.13(2.83)$ & 0.1974 \\
\hline Device SimK ave & $42.68(3.86)$ & $42.42(3.38)$ & 43.57 (3.59) & 0.1900 \\
\hline Calc SimK steep & $44.89(4.40)$ & 44.81 (4.09) & $45.70(4.83)$ & 0.3608 \\
\hline Calc SimK flat & $40.74(3.75)$ & 41.35 (3.51) & $42.17(3.54)$ & 0.2826 \\
\hline Calc SimK ave & $42.82(4.01)$ & $43.08(3.72)$ & $43.93(4.12)$ & 0.4346 \\
\hline Kmax & $50.83(4.46)$ & $52.64(4.21)$ & $52.50(5.06)$ & 0.0169 \\
\hline CLMI_axi & $12.38(4.16)$ & $14.61(4.12)$ & $14.02(4.52)$ & 0.2024 \\
\hline CLMI_Spotaxi & $50.01(4.42)$ & $51.22(4.03)$ & $51.37(4.91)$ & 0.2430 \\
\hline PCLMI_axi & $-2.66(0.56)$ & $-3.03(0.75)$ & & 0.2242 \\
\hline PCLMI_Spotaxi & $-7.85(0.66)$ & $-8.17(0.60)$ & & 0.0880 \\
\hline
\end{tabular}


a cross-linking procedure. Interestingly, the OSUCTTcalculated SimKs followed the same pattern as other anterior parameters, with the small-cone Placido having the highest values and the pure Scheimpflug the lowest. However, this pattern disappeared in the native SimKs reported by the devices. The relationship between measured SimKs reflects the differences in the algorithms of each device for simulating manual keratometry. Again, it is not possible with the current study design to determine which device produced a SimKs that were closest to Ks from manual keratometry. The differences and similarities between the three technologies in measurements of the same subject are shown in Figures 4A and B which shows the native device and the corresponding displays from OSUCTT using the same scales and display features.

Kmax is a single point measurement, compared to CLMI_spot which is an average over the $2 \mathrm{~mm}$ diameter region of greatest curvature. As an average, one might expect CLMI_spot to show less variability than a single point measurement, which was demonstrated in the current study, with a larger standard deviation and a higher mean value in Kmax than in CLMI_spot. In addition, it has been reported that the precision of single point measurements on corneal maps is poor. ${ }^{20}$ Thus, one might also expect CLMI_spot to be more robust than Kmax in evaluating response to treatment, such as corneal collagen cross-linking. This will require further study by investigating post-cross-linking data overtime.

CLMI_axi has been found to be an accurate indicator of keratoconus in a series of known keratoconic eyes. This study demonstrates that while there are significant differences, although clinically not relevant, with the measurements between specific topographic/tomographic instruments (Keratron compared to Galilei and Pentacam), the CLMI values are still predictive of the diagnosis of keratoconus.

This study presents CLMI_axi values for a small set of postrefractive ectasia eyes. The small $\mathrm{n}$ is likely underpowered to detect differences between devices. However, these eyes have simulated keratometric values in the normal range (Ave $\mathrm{K}$ of 42.68, 42.42 and 43.56 diopters respectively, for Galilei, Pentacam and Keratron instruments), but the CLMI_axi values are similar or higher than those of keratoconus, 12.39, 14.61 and 14.01 diopters respectively, indicating substantial asymmetry in surface shape. CLMI calculated from axial data in normal eyes has been reported at $0.95(n=78)$, compared to $8.66(n=25)$ for keratoconic eyes. ${ }^{14}$ Early diagnosis of keratoconus and postrefractive ectasia has become crucial with the emergence of corneal collagen cross-linking treatments which may slow, halt or reverse the progression of the disease. ${ }^{26}$ CLMI may prove of greater clinical utility than SimKs in the case of postrefractive ectasia.

The posterior CLMI (PCLMI_axi) is reported for each group. There have been no published data on normal eyes for PCLMI_axi. The values are -2.46 and -2.60 diopters for the Galilei and Pentacam respectively, for keratoconic corneas -2.66 and -3.04 diopters for the ectasia corneas using axial data. Converting the posterior dioptric values to account for the refractive index difference in order to compare with the anterior CLMI, the values would be 20.76 and 21.94 diopters for keratoconus, 22.44 and 25.65 diopters for ectasia respectively. This compares to the anterior values of 11.20, 11.00, 12.39 and 14.61 diopters for the axial CLMI on the Galilei and the Pentacam for the keratoconic and ectasia eyes respectively. The converted posterior CLMI is on average almost twice (1.84) the magnitude of the anterior CLMI, indicating that the physical size of the asymmetry on the posterior surface is greater than that on the anterior surface. This is illustrated in Figure 5, which shows the anterior and posterior best-fit sphere (BFS) elevations from the same subject as Figures $4 \mathrm{~A}$ and $\mathrm{B}$. Note that the average curvature of the anterior surface is less (BFS radius $=7.41 \mathrm{~mm}$ ) than that of the posterior surface (BFS radius $=6.10 \mathrm{~mm}$ ). From these maps, it can be easily appreciated that the maximum elevation on the posterior surface is larger physically than the corresponding feature on the anterior surface. However, the magnitude of the greater posterior surface asymmetry is buried in the conversion to diopters due to the much smaller difference in index of refraction between the cornea and aqueous at the posterior surface, compared to the larger difference between air and cornea on the anterior surface. This deserves further evaluation to determine, if it could be a more sensitive measure of early ectasia and keratoconus. ${ }^{27-29}$

\section{CONCLUSION}

The cone location and magnitude index (CLMI) may be calculated using data from various topographic and tomographic devices. Although the different technologies may yield significantly different results, the magnitude of the differences are likely not clinically relevant in the management of ectatic disorders. The small-cone Placido tends to produce larger dioptric values on the order of about 1 diopter in keratoconus. The pure Scheimpflug and combined Scheimpflug/Placido devices are more similar in their measured values. The CLMI calculation remains valuable in predicting keratoconus from the observed data. Posterior CLMI may offer another valuable measure of 

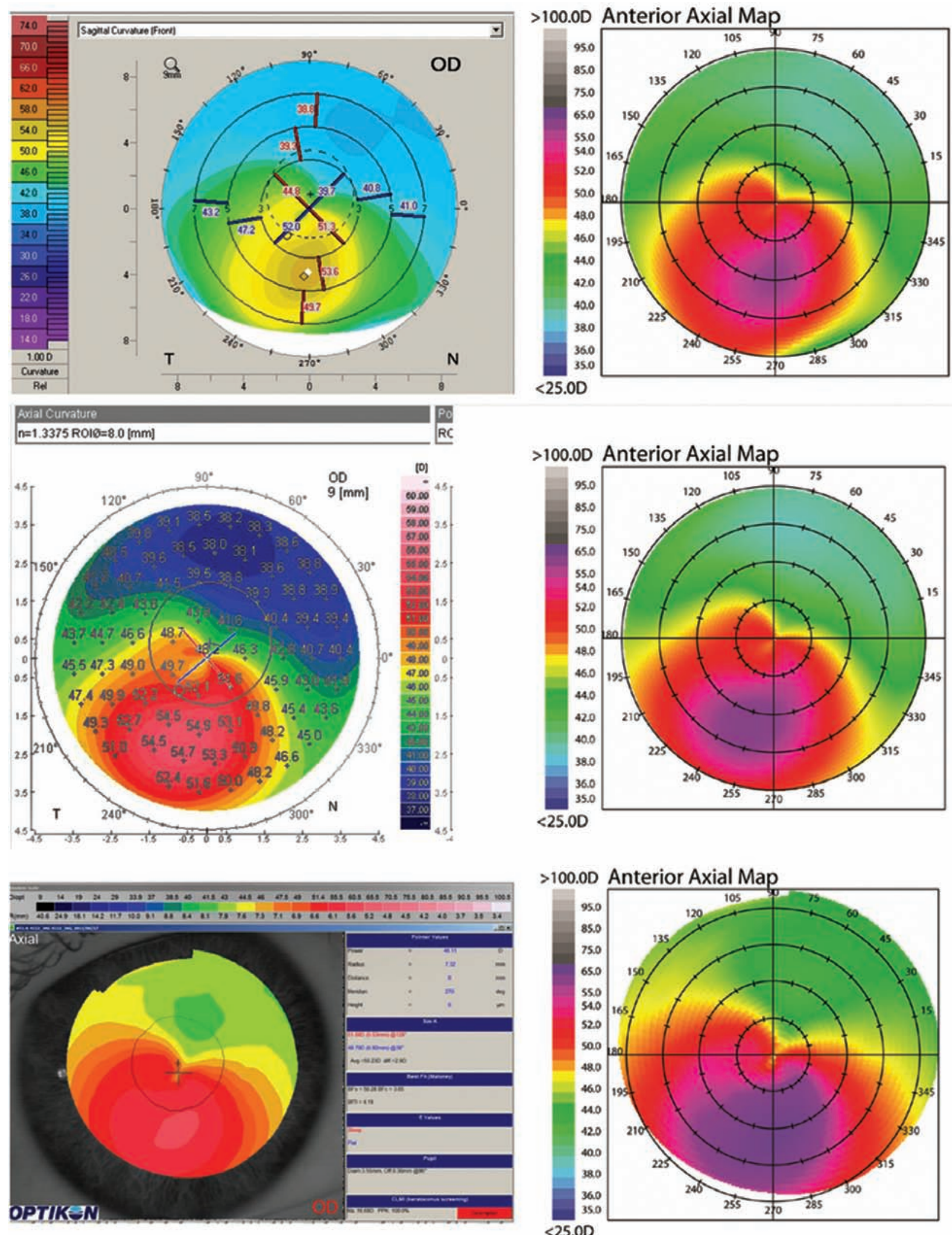

Fig. 4A: Comparison of native device display of anterior axial maps from pure Scheimpflug, combination Scheimpflug/Placido and pure Placido systems (upper, middle and lower respectively) and the corresponding OSUCTT maps which use the same display features and color scale. Note the similarities between systems in the OSUCTT displays that are more difficult to appreciate in the native displays

asymmetric steepening of the cornea. Baseline calculations in normal eyes are necessary to validate the posterior CLMI for keratoconic and postrefractive surgery ectasia eyes.
Stratification of disease and monitoring for small amounts of progression overtime remain challenges in the management of these diseases. ${ }^{30}$ 

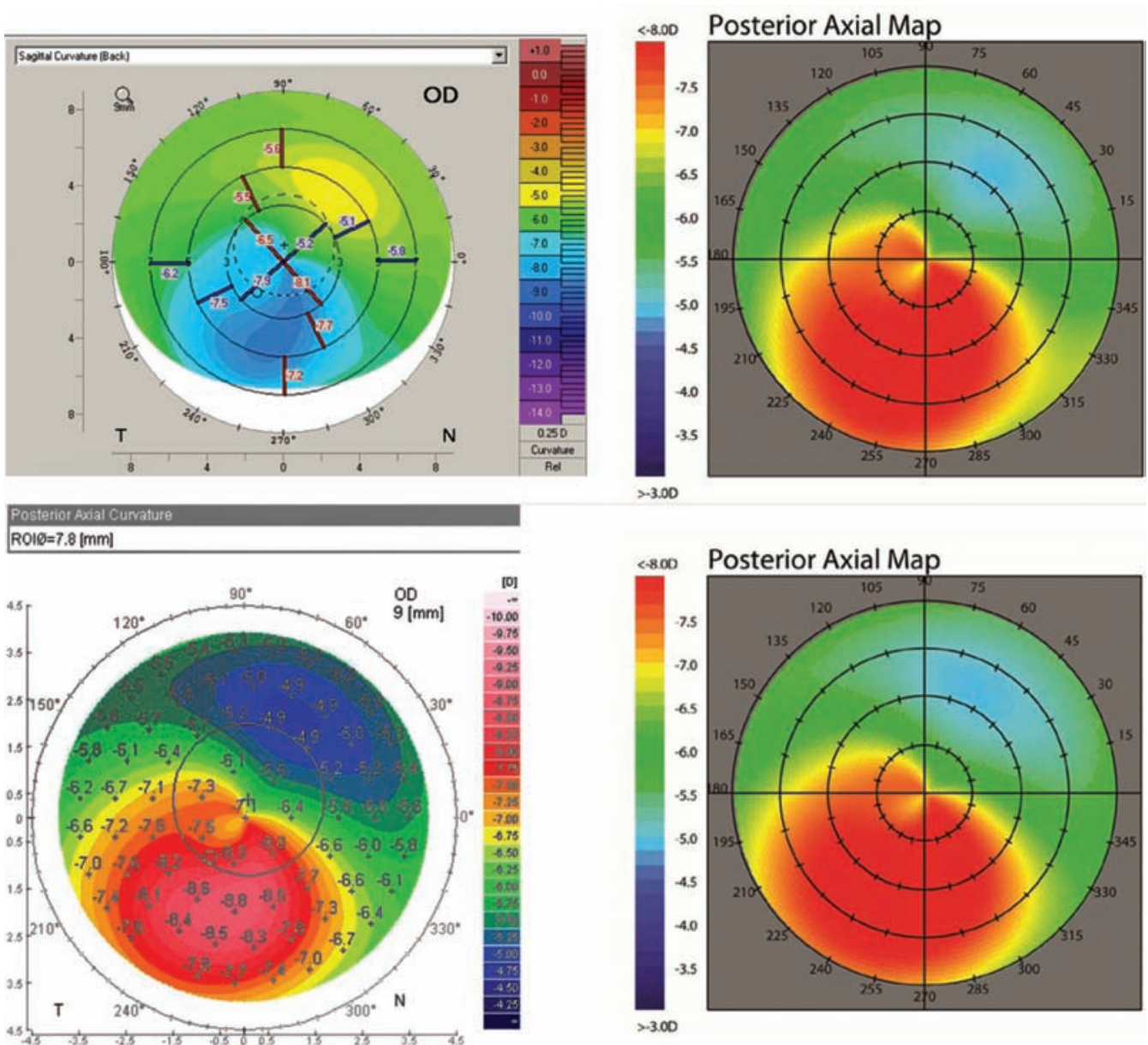

Fig. 4B: Comparison of native device displays of posterior axial maps from pure Scheimpflug and combination Scheimpflug/Placido (upper and lower respectively), compared to standard OSUCTT displays. Note that the pure Scheimpflug system shows the largest negative dioptric values in blue which correspond to the greatest curvature on the posterior surface. The OSUCTT display uses red to correspond to the greatest curvature values on the posterior surface which in this case are greater values in the negative direction

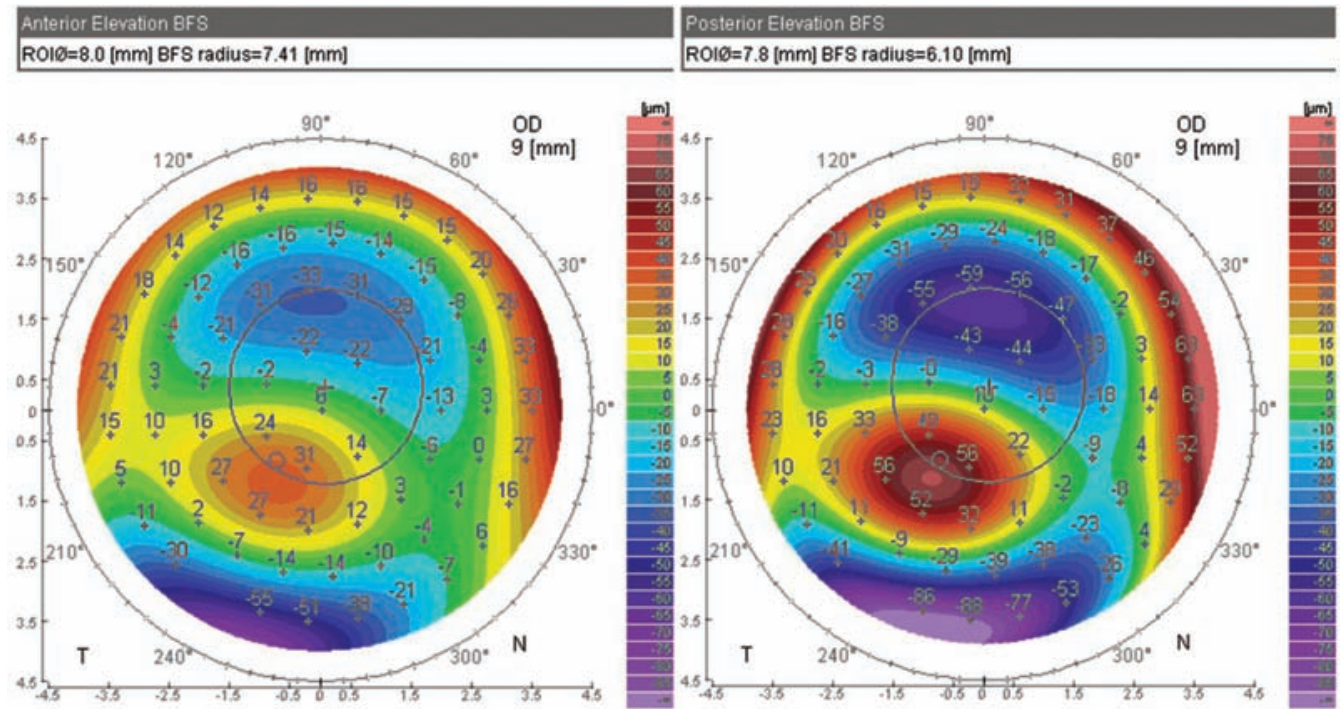

Fig. 5: Best-fit sphere (BFS) elevation maps from the anterior surface (left) and the posterior surface (right), from the same subject as shown in Figures $4 A$ and $B$, illustrating the physically larger maximum elevation on the posterior surface compared to the anterior surface

\section{REFERENCES}

1. Krachmer JH, Feder RS, Belin MW. Keratoconus and related noninflammatory corneal thinning disorders. Surv Ophthal 1984;28:293-322.
2. Belin MW, Asota IM, Ambrosio R, Khachikan SS. What's in a name? Keratoconus, Pellucid marginal degeneration and related thinning disorders. Am J Ophthalmol 2011;152:157-62.

3. Belin MW, Khachikian SS. Keratoconus: It is hard to define, but ... . Am J Ophthalmol 2007;143:500-03. 
4. Twa MD, Nichols JJ, Joslin CE, et al. Characteristics of corneal ectasia after LASIK for myopia. Cornea 2004;23:447-57.

5. Seiler T, Quurke AW. Iatrogenic keratectasia after LASIK in a case of forme frusta keratoconus. J Cataract Refract Surg 1998;24:1007-09.

6. Randleman JB, Russel B, Ward M, et al. Risk factors and prognosis for corneal ectasia after LASIK. Ophthalmology 2003;110:267-75.

7. Binder PS, Lindstrom RL, Stulting RD, et al. Keratoconus and corneal ectasia after LASIK. J Cataract Refract Surg 2005;35: 2035-38.

8. Maeda N, Klyce SD, Smolek MK, et al. Automated keratoconus screening with corneal topography analysis. Invest Ophthalmol Vis Sci 1994;35:2749-57.

9. Maeda N, Klyce SD, Smolek MK. Neural network classification of corneal topography. Preliminary demonstration. Invest Ophthalmol Vis Sci 1995;36:1327-35.

10. Rabinowitz YS. Videokeratographic indices to aid in screening for keratoconus. J Refract Surg 1995;11:371-79.

11. Savini G, Carbonelli M, Sbreglia A, Baroni P, Deluigi G, Hoffer $\mathrm{K}$. Comparison of anterior segment measurements by 3 Scheimpflug tomographers and 1 Placido corneal topographer. J Cataract Refract Surg 2011;37:1679-85.

12. Mahmoud AM, Roberts C, Henderick EE. The Ohio State University Corneal Topography Tool. Invest Ophthalmol Vis Sci 2000;41:S677.

13. Mahmoud A, Roberts C, Herderick EE, et al. The cone location and magnitude index (CLMI). Invest Ophthalmol Vis Sci 2001;42:S4828.

14. Mahmoud AM, Roberts CJ, Lembach RG, Twa MD, Herderick EE, McMahon TT. CLEK study group. CLMI the cone location and magnitude index. Cornea 2008;27:480-87.

15. Mahmoud A, Roberts C, Lembach R, et al. Simulation of machine-specific topographic indices for use across platforms. Optom Vis Sci 2006;83:682-93.

16. Oshika T, Tomidokoro A, Maruo K, et al. Quantitative evaluation of irregular astigmatism by Fourier series harmonic analysis of videokeratography data. Invest Ophthalmol Vis Sci 1998; 39:705-09.

17. Twa MD, Parthasarathy S, Roberts C, Mahmoud AM, Raasch TW, Bullimore MA. Automated decision tree classification of corneal shape. Optometry and Vision Science, December 2005; 82(12):1038-46.

18. Karon MD, Klyce SD, Smolek MK. Device-independent statistical indexes in corneal topography. Invest Ophthalmol Vis Sci 2004;45 E-abstract 2874.

19. Rabinowitz YS, Rasheed K. KISA\% index: A quantitative videokeratography algorithm embodying minimal topographic criteria for diagnosing keratoconus. J Cataract Refract Surg 1999;25:1327-35.

20. McAlinden C, Khadka J, Pesudovs K. A comprehensive evaluation of the precision (repeatability and reproducibility) of the Oculus Pentacam HR. Investigative Ophthalmology and Visual Science 2011;52(10):7731-37.

21. Shankar H, Taranath D, Santhirathelagan CT, Pesudovs K. Anterior segment biometry with the Pentacam: Comprehensive assessment of repeatability of automated measurements. Journal of Cataract and Refractive Surgery 2008;34(1):103-13.

22. Kawamorita T, Nakayama N, Uozato H. Repeatability and reproducibility of corneal curvature measurements using the Pentacam and Keratron topography systems. Journal of Refractive Surgery 2009;25(6):539-44.
23. Menassa N, et al. Comparison and reproducibility of corneal thickness and curvature readings obtained by the Galilei and the Orbscan II analysis systems. Journal of Cataract and Refractive Surgery 2008;34(10):1742-47.

24. Savini G, Carbonelli M, Barboni P, Hoffer KJ. Repeatability of automatic measurements performed by a dual Scheimpflug analyzer in unoperated and postrefractive surgery eyes. Journal of Cataract and Refractive Surgery 2011;37(2):302-09.

25. Tripoli NK, Cohen KL, Holmgren DE, Coggins JM. Assessment of radial aspheres by the Arc-step algorithm as implemented by the Keratron keratoscope. American Journal of Ophthalmology 1995;120(5):658-64.

26. Vinciguerra P, Albè E, Trazza S, Seiler T, Epstein D. Intraoperative and postoperative effects of corneal collagen cross-linking on progressive keratoconus. Arch Ophthalmol Oct 2009;127(10):1258-65.

27. Wilson SE. Cautions regarding measurement of the posterior corneal curvature. Ophthalmology 2000;107:1223.

28. Tomidokoro A, Oshika T, Amano S, et al. Changes in anterior and posterior corneal curvatures in keratoconus. Ophthalmology 2000;107:1328-32.

29. Pinero DP, Saenz Gonzales C, Ali_o JL. Intraobserver and interobserver repeatability of curvature and aberrometric measurements of the posterior corneal surface in normal eyes using Scheimpflug photography. J Cataract Refract Surg 2009; 35:113-20.

30. Maguire LJ, Lowry JC. Identifying progression of subclinical keratoconus by serial topography analysis. Am J Ophthalmol 1991;112:41-45.

\section{ABOUT THE AUTHORS}

\section{Thomas F Mauger}

Chairman, Department of Ophthalmology, The Ohio State University, Ohio, USA

\section{Ashraf M Mahmoud}

System Analyst, Department of Ophthalmology, The Ohio State University, Ohio, USA

\section{Cynthia J Roberts (Corresponding Author)}

Professor, Department of Ophthalmology, The Ohio State University, Ohio, USA, e-mail: roberts.8@osu.edu

\section{Lena V Chheda}

Assistant Professor, Department of Ophthalmology, The Ohio State University, Ohio, USA

\section{Rebecca A Kuennen}

Assistant Professor, Department of Ophthalmology, The Ohio State University, Ohio, USA

\section{Andrew J Hendershot}

Assistant Professor, Department of Ophthalmology, The Ohio State University, Ohio, USA

\section{Richard G Lembach}

Professor, Department of Ophthalmology, The Ohio State University Ohio, USA 